Mots. Les langages du politique

\title{
Justifier ou illégitimer la peine de mort ? Aspects argumentatifs du débat parlementaire de 1981
}

For or against capital punishment: argumentative aspects of the 1981 French parliamentary debate

¿ Justificar o desligitimar la pena de muerte? Aspectos argumentativos del debate parlamentario francés de 1981

\section{Raphaël Micheli}

\section{OpenEdition}

Journals

Édition électronique

URL : https://journals.openedition.org/mots/4903

DOI : $10.4000 /$ mots.4903

ISSN : 1960-6001

Éditeur

ENS Éditions

\section{Édition imprimée}

Date de publication : 1 mars 2004

Pagination : 109-121

ISBN : 2-84788-056-9

ISSN : 0243-6450

\section{Référence électronique}

Raphaël Micheli, « Justifier ou illégitimer la peine de mort ? Aspects argumentatifs du débat parlementaire de 1981 », Mots. Les langages du politique [En ligne], 74 | 2004, mis en ligne le 24 avril 2008, consulté le 22 avril 2022. URL : http://journals.openedition.org/mots/4903 ; DOI : https:// doi.org/10.4000/mots.4903 


\section{Justifier ou illégitimer la peine de mort? Aspects argumentatifs du débat parlementaire de $1981^{2}$}

Le débat sur la peine de mort a trouvé son issue en France, après plus de deux siècles, lors du vote de l'abolition par l'Assemblée nationale et par le Sénat au mois de septembre 1981. S'il semble aujourd'hui relativement bien connu, en raison du grand renom de certains de ses protagonistes et de la place qu'il s'est récemment vu octroyer dans les médias ${ }^{3}$, ce débat n'a cependant pas encore été étudié sous un jour proprement discursif. C'est précisément à ce niveau que j'entends situer la spécificité de ma recherche, en examinant quelques extraits tirés des comptes rendus de la session parlementaire extraordinaire de septembre 1981. La question à laquelle je souhaite apporter des éléments de réponse se laisse formuler ainsi : quelles logiques et quels types de légitimité sous-tendent les diverses positions discursives adoptées face à la peine capitale? Ma réflexion s'inscrit à la fois dans l'analyse des pratiques discursives et dans le renouveau des études rhétoriques au $20^{\mathrm{e}}$ siècle.

1. Centre de recherches en linguistique textuelle et en analyse des discours, Université de Lausanne, CH1015 Dorigny (Raphael.Micheli@frmod.unil.ch)

2. Je remercie Marc Dominicy et Emmanuelle Danblon (Université libre de Bruxelles), ainsi que Jean-Michel Adam et Thierry Herman (Université de Lausanne) pour leur aide précieuse.

3. Le vingtième anniversaire de l'abolition a vu la réédition en masse des textes abolitionnistes les plus fameux, phénomène encore accentué, en 2002, par le bicentenaire de la naissance de Victor Hugo. Ce regain d'intérêt éditorial pour la question de la peine de mort ne s'est pas limité au seul domaine littéraire. Voir S. Costa (éd.), 2001, La peine de mort; de Voltaire à Badinter, Paris, Garnier Flammarion, pour un recueil de textes abolitionnistes relevant de genres discursifs divers; ou encore F. Johannès, C. Prieur (éd.), 2002, La peine de mort, Paris, Librio, pour un recueil des articles du quotidien Le Monde relatifs à la peine capitale. La perspective adoptée par les éditeurs de ces publications privilégie l'intérêt historique du débat sur la peine de mort. Si l'on assiste parfois à l'énumération des arguments les plus marquants des parties en présence, on manque d'une analyse qui envisagerait ces schèmes argumentatifs dans leurs multiples réalisations langagières et dans leurs agencements complexes au sein de textes particuliers. 
L'analyse des discours, telle qu'elle se développe dans le monde francophone depuis plus de trente ans, cherche essentiellement à étudier «l'intrication d'un mode d'énonciation et d'un lieu social déterminé» ${ }^{4}$. Il s'agit, en d'autres termes, de relier l'étude de configurations textuelles particulières à une compréhension plus large des pratiques discursives dans lesquelles ces configurations font sens. Devant un corpus donné, l'analyste du discours part ainsi à la recherche de «régularités» qui ne tiennent pas uniquement aux contraintes exercées par la langue «comme code transcendant la diversité sociale de ses pratiques $\gg^{5}$ : il s'attache également à décrire l'effet des contraintes exercées par la situation d'interaction et par le(s) genre(s) discursif(s) $\mathrm{au}(\mathrm{x})$ quel(s) le texte s'indexe plus ou moins conflictuellement. Dans le cas qui m'intéresse, le Parlement doit être pensé comme une institution qui soumet la prise de parole individuelle à un ensemble de règles et de contraintes. Comme le suggèrent les juristes Pierre Avril et Jean Gicquel, «l'unité des activités par lesquelles les assemblées remplissent leurs différentes fonctions résulte de ce qu'elles s'accomplissent sur le mode de la délibération $»^{6}$. Si une telle affirmation tend à laisser de côté certaines des pratiques discursives parlementaires non officielles - comme les petites phrases lancées à la presse par les députés les plus expérimentés au sortir de l'hémicycle -, il me semble qu'elle s'applique assez bien à la discussion générale d'un projet de loi, d'où sont tirés mes exemples.

Mais comment caractériser ce «mode de la délibération»? On peut penser ici au «genre» délibératif de la rhétorique aristotélicienne. Si ce dernier ne saurait constituer une catégorie assez fine pour appréhender les pratiques discursives du Parlement moderne, il peut néanmoins être d'un rendement descriptif certain dans le cadre de mon travail. On peut le concevoir non comme une catégorie macrotextuelle, mais, plus modestement, comme un ensemble de traits - que ces derniers soient d'ordre sémantique, inférentiel-argumentatif ou, plus largement, rhétorique. La présence plus ou moins marquée de tels traits conduit à caractériser les discours de manière graduelle, en termes de dominante. L'essentiel, pour saisir ce qui distingue les discours abolitionnistes des discours antiabolitionnistes, est de comprendre que l'indexation des différents orateurs à ce «mode de la délibération» peut s'avérer fort problématique. Elle va même jusqu'à donner lieu à des affrontements sur ce que Jean-Michel Adam

\footnotetext{
4. D. Maingueneau, (dir.), 1995, «Introduction», Les analyses de discours en France, Langages, $\mathrm{n}^{\circ} 117$, Paris, Larousse, p. 7-8.

5. M. Angenot, 1997, La propagande socialiste. Six essais d'analyse du discours, Montréal, LUnivers du Discours, p. 29-30.

6. P. Avril, J. Gicquel, 1996, Droit parlementaire, $2^{\mathrm{e}}$ édition, Paris, Montchrestien, p. 127-128.
} 
appelle «les façons légitimes de parler au sein d'une formation socio-discursive donnée $»^{7}-$ dans mon cas, sur les façons légitimes d'argumenter pour ou contre l'abolition de la peine de mort au sein de l'institution parlementaire. L'hypothèse, à ce titre, est la suivante: les discours antiabolitionnistes se caractérisent par une forte dominante délibérative, ce qui n'est en revanche pas le cas des discours abolitionnistes.

Pour mettre cette hypothèse à l'épreuve, j'examinerai successivement trois extraits du débat parlementaire de 1981, dont deux sont particulièrement représentatifs de l'argumentation antiabolitionniste et le troisième emblématique de l'argumentation abolitionniste. Les deux interrogations suivantes guideront mon analyse: s'agit-il, pour les orateurs, de qualifier l'objet de la délibération selon l'opposition utile/nuisible ou plutôt selon l'opposition juste/injuste? En d'autres termes, faut-il envisager prioritairement les conséquences pratiques de la décision parlementaire ou faut-il remonter vers ses fondements éthiques?

\section{L'utilité des conséquences: deux exemples d'argumentation antiabolitionniste}

Les extraits (1) et (2) sont tirés d'interventions de parlementaires antiabolitionnistes. Pour brefs qu'ils soient, ils permettent de saisir et d'exemplifier la dynamique d'une argumentation antiabolitionniste prototypique ${ }^{8}$ :

(1) Quelles que soient les circonstances, le droit à la vie se doit d'être respecté, et plus la victime est faible et sans défense, plus elle mérite d'être défendue.

Voilà le nœud du problème: quel que soit le degré de responsabilité du criminel, quelle est la vie la plus précieuse à défendre et qu'il importe le plus de sauver: l'assassin odieux ou l'innocente victime, non pas seulement celle qui vient de perdre la vie souvent dans des circonstances cruelles, mais aussi toutes les innocentes victimes en puissance? [...]

L'État doit se préoccuper des victimes et de la sécurité des citoyens plutôt que d'assurer l'avenir des criminels (Marcel Bigeard, AN, p. 1164).

7. J.-M. Adam, 1999, Linguistique textuelle. Des genres de discours aux textes, Paris, Nathan, p. 91.

8. Les extraits sont tirés des comptes rendus intégraux des séances des 17 et 18 septembre 1981 à l'Assemblée nationale, publiés dans le Journal Officiel de la République française, p. 1136-1223, ainsi que des comptes rendus intégraux des séances des 28, 29 et 30 septembre 1981 du Sénat, publiés dans le Journal Officiel de la République française, p. 1662-1749. J'adopterai désormais le système de références suivant: nom de l'orateur, lieu institutionnel (AN : Assemblée nationale et S: Sénat) et numéro de la page du Journal Officiel d'où est tiré l'extrait choisi. 
(2) D'abord, permettez-moi de souligner l'intérêt que j'attache à la vie, à toutes les vies humaines - fussent-elles celles de criminels - ce qui m'interdit toute légèreté, toute faiblesse et toute passion dans la présente intervention. Nous voici donc en présence d'intérêts contradictoires qui nous mettent dans la douloureuse obligation de choisir entre la vie d'un assassin parfois récidiviste, souvent irrécupérable, et celles d'innocentes victimes aveuglément menacées. Ce choix me semble facile: c'est d'abord l'élément le plus sain de la société qu'il faut protéger. [...]

[J']avoue que je comprends la démarche [des abolitionnistes], sans trouver leurs arguments convaincants au seul et suffisant motif qu'ils favorisent des intérêts minoritaires par rapport à d'autres beaucoup plus importants et indiscutablement plus respectables. (Jean Amelin, S, p. 1700)

\section{Une prémisse encombrante: le droit à la vie}

Les extraits s'ouvrent tous deux sur l'assertion d'une prémisse apparemment partagée avec l'adversaire, celle du droit à la vie. Cette prémisse s'appuie implicitement sur des sources accréditées au sein de l'institution parlementaire - les dix commandements de l'Ancien Testament et, dans une perspective laïque, les droits de l'homme hérités de la Révolution française. L'affirmation d'une telle prémisse tend ainsi, dans un premier temps, au renforcement des croyances qui assurent à l'auditoire sa cohésion et à l'atténuation de la distance entre les groupes antagonistes. On est ici proche de ce que les Anciens appelaient l'homonoia, une communauté de sentiments qui, à ce stade de l'argumentation, transcende encore les clivages politiques. Si les orateurs de (1) et (2), comme la majorité de leurs collègues antiabolitionnistes, exhibent fièrement leur adhésion au principe du droit à la vie, la portée dudit principe ne va cependant pas sans quelque ambigüité. Un conflit se fait jour entre la portée théoriquement universelle du principe et une grande difficulté à appliquer ce que Chaïm Perelman appelle la règle de justice formelle: «Les êtres d'une même catégorie essentielle doivent être traités de la même façon ${ }^{9}$. L'orateur de l'extrait (1) s'exprime en ces termes: "Quelles que soient les circonstances, le droit à la vie se doit d'être respecté, et plus la victime est faible et sans défense, plus elle mérite d'être défendue». Ici, le tour universalisant de la circonstancielle se voit contrecarré par la proposition qu'introduit le et. Le tour graduel de cette proposition laisse penser qu'il va s'agir, pour les orateurs antiabolitionnistes, de hiérarchiser les différents groupes d'individus auxquels s'applique le principe du droit à la vie et de restreindre ainsi son champ d'application. On ne va pas assister à un rejet pur et simple de la prémisse partagée, difficilement envisageable compte tenu de

9. C. Perelman, 1977, L'empire rhétorique, Paris, Vrin, p. 81. 
la doxa qui rend l'échange parlementaire possible, mais bien à une périlleuse recherche de compatibilité.

\section{Restriction du champ d'application et dissociation de la notion}

Pour échapper à l'inférence qui découle directement de l'assertion de la prémisse selon laquelle «tout individu a droit à la vie» (selon la formulation de la Déclaration universelle des droits de l'homme de 1948), les orateurs antiabolitionnistes doivent mettre l'accent sur un conflit de règles. Le conflit nait lorsque "protéger la vie de l'assassin» a pour conséquence d' «exposer celle de l'innocent», selon la formule lapidaire d'un autre député antiabolitionniste (Serge Charles, AN, p. 1174). Dans une telle situation, l'application de la première règle - le respect strict du droit à la vie - contrevient à l'application de la seconde règle selon laquelle «la société $[\ldots]$ a $[\ldots]$ le devoir de se protéger et de protéger les individus qui la composent» (Gilbert Gantier, $\mathrm{AN}$, p. 1992). L'incompatibilité présumée entre ces deux règles conduit les orateurs à restreindre le champ d'application de la première règle et à opérer ce que C. Perelman appelle une «division quant à l'objet» ${ }^{10}$ auquel cette dernière s'applique in abstracto.

Par un processus de binarisation, l'ensemble des individus dénotés par le syntagme «toutes les vies humaines» se voit scindé en deux groupes. L'usage de tours superlatifs - par exemple, en (1), «la vie la plus précieuse à défendre » et en (2) l'«élément le plus sain qu'il faut protéger» - suggère que l'un de ces deux groupes va se voir isolé comme le premier de l'ensemble: la binarisation s'accompagne bien d'une hiérarchisation. Dans nos extraits, on a, d'un côté, l' «assassin odieux» (1), "parfois récidiviste, souvent irrécupérable» (2), et, de l'autre, les «innocentes victimes» (2). On constate que la qualification des individus tend à une sorte de fixation ontologique. Dans les discours antiabolitionnistes, il ne suffit pas qu'un homme ait commis un meurtre, il faut qu'il devienne par essence un meurtrier. Dans cet esprit, les adverbes et adjectifs épithètes utilisés ne font que renforcer la connotation intrinsèquement négative du lexème «assassin». On sera également attentif au syntagme «innocente[s] victime[s] » qui revient à trois reprises dans nos extraits. Dans ce syntagme, l'antéposition de l'adjectif interdit à ce dernier de se voir suivi d'un complément: la victime n'est alors plus innocente de quelque chose. Le fait stylistique d'antéposition et le figement du syntagme font de l'innocence non plus une propriété relative à une circonstance ponc-

10. C. Perelman, L. Olbrechts-Tyteca, 2000 ( $1^{\text {re }}$ édition 1958), Traité de l'argumentation, Bruxelles, Éditions de l'Université de Bruxelles, p. 271. 
tuelle, mais bien une propriété essentielle de l'individu décrit. Le second trait marquant de la qualification des victimes est l'insistance sur leur supériorité numérique. L'orateur de (1) ne se contente pas d'opposer de manière symétrique l'《assassin odieux» à l'«innocente victime»: la binarisation s'étend jusqu'à «toutes les innocentes victimes en puissance» - tout comme en (2) où l'orateur oppose "la vie d'un assassin» à «celles d'innocentes victimes aveuglément menacées ». Du point de vue du nombre, il y a une très nette asymétrie entre les deux groupes d'individus placés en concurrence pour l'octroi du droit à la vie.

Le processus de binarisation que je viens de décrire permet de restreindre le champ d'application du droit à la vie. Une telle restriction se justifie lorsque le droit à la vie de certains individus peut exercer des conséquences néfastes sur le droit à la vie d'autres individus qui, en plus d'être moralement irréprochables, sont plus nombreux! Il me semble ainsi que la restriction comporte deux versants: l'un éthique - l'orateur de (2) parle d'intérêts «indiscutablement plus respectables»-, l'autre, plus typiquement délibératif, se rapportant à l'intérêt général - le même orateur souligne le danger de «favoris[er] des intérêts minoritaires ». Dans les deux cas, on considère le droit à la vie en aval, en envisageant les conséquences directes de son application.

Cette double restriction permet de ne pas tomber sous le coup de l'inférence immédiate de la prémisse qui asserte le droit à la vie de chaque individu «quelles que soient les circonstances». Dans une optique antiabolitionniste, l' «assassin odieux» ne saurait avoir la vie sauve pour la seule raison qu'il appartient à la même «catégorie essentielle» - celle des êtres humains - que les «innocentes victimes». Les trajets argumentatifs considérés partent bien de la notion de droit à la vie, mais, devant le conflit de règles, opèrent une dissociation de cette notion. C. Perelman affirme que la dissociation d'une notion est l'une des techniques argumentatives les plus efficaces - sinon la plus efficace - pour «lever une incompatibilité $»^{11}$. Le travail de dissociation implique, de manière cruciale, une hiérarchisation au terme de laquelle apparaissent un pôle supérieur et un pôle inférieur de la notion considérée ${ }^{12}$. Dans le cas qui nous intéresse, le pôle inférieur s'apparente, on s'en souvient, au respect unilatéral du droit à la vie, abstraction faite des conséquences possibles. La pôle supérieur, en revanche, équivaut à une application restreinte qui tienne compte de la qualité éthique des personnes considérées et surtout, dans une perspec-

11. C. Perelman, L. Olbrechts-Tyteca, 2000, ouvr. cité, p. 552 et suiv.

12. M. Dominicy, 2002, «La dimension sémantique du discours argumentatif: le travail sur les notions», dans R. Amossy, R. Koren (dir.), Après Perelman : quelles politiques pour les nouvelles rhétoriques?, Paris-Montréal, L'Harmattan, p. 123-147. 
tive délibérative, de leur caractère minoritaire ou majoritaire. Par le coup de force argumentatif que constitue la dissociation hiérarchisée, les orateurs antiabolitionnistes peuvent prétendre venir à bout d'un paradoxe: l'attachement à la peine capitale n'irait pas forcément de pair avec un renoncement au respect de la vie humaine. Selon eux, la notion floue de «droit à la vie», si elle est bien comprise in situ, concerne avant tout la majorité «respectabl[e]» de la Cité. Dans un tel ordre d'idées, la peine capitale respecte le droit à la vie, ou plutôt le pôle supérieur de cette notion que le travail de dissociation a eu pour tâche de dégager. L'argumentation antiabolitionniste ne se débarrasse ainsi pas purement et simplement d'une prémisse que j'ai qualifiée d'encombrante; elle vise plutôt, par la dissociation des notions, à rendre cette prémisse compatible avec la thèse défendue.

\section{Une légitimité typiquement délibérative}

Les orateurs antiabolitionnistes se mettent constamment en scène comme des «législateurs» et comparent leurs adversaires abolitionnistes à des «philosophes », dans une acception péjorative de ce terme. Une telle opposition nous ramène à la question du genre ou, à tout le moins, du mode approprié au sein de l'institution parlementaire et du combat pour la légitimité. Selon les orateurs antiabolitionnistes, la réponse parlementaire à la question «Faut-il abolir la peine de mort?» ne saurait se baser sur la seule assertion d'un principe supposé intangible. Dans une optique délibérative, elle doit au contraire établir que la décision parlementaire, de par ses conséquences pratiques, concourra uniformément au bonheur de la Cité. Si l'assertion d'une notion floue et abstraite de tout contexte peut convenir à un philosophe, un orateur délibératif tel qu'un législateur ne saurait en tirer aucune inférence immédiate avant d'avoir jaugé son application en contexte et son caractère utile ou nuisible pour la collectivité. La légitimité des trajets argumentatifs étudiés ici dépend étroitement d'une dominante délibérative supposément valorisée au sein du Parlement lors de la discussion générale d'un projet de loi.

\section{À la recherche de fondements justes: un exemple d'argumentation abolitionniste}

La rapide étude du discours antiabolitionniste que je viens de livrer montre que celui-ci repose avant tout sur une argumentation projective où sont prioritairement envisagées les conséquences de la thèse qui fait débat. Les orateurs abolitionnistes, en revanche, envisagent la délibération d'une tout autre manière. Pour eux, il s'agit moins, selon l'expression de C. Perelman, 
d'«apprécier la décision en fonction [...] [de son] efficacité et [de son] opportunité» que de $l^{\prime}$ «encadre[r] dans un contexte de valeurs et de normes $»^{13}$. Partant de l'idée que leurs adversaires accordent à la société «le droit d'ôter la vie à l'un de ses membres» (Pierre Vallon, S, p. 1703), les abolitionnistes vont se pencher sur ce qui prétend fonder ce droit. Je considérerai l'extrait suivant, tiré du discours du garde des Sceaux à l'Assemblée nationale:

(3) La vérité est qu'au plus profond des motivations de l'attachement à la peine de mort, on trouve, inavouée le plus souvent, la tentation de l'élimination [...] L'assassin doit mourir tout simplement parce que, ainsi, il ne récidivera pas. Et tout parait si simple, et tout parait si juste.

Mais quand on accepte ou que l'on prône la justice d'élimination, au nom de la justice, il faut bien voir dans quelle voie on s'engage. Pour être acceptable, même pour ses partisans, la justice qui tue le criminel doit tuer en connaissance de cause [...]. Il s'agit bien, en définitive, dans l'abolition, d'un choix fondamental, d'une certaine conception de l'homme et de la justice. Ceux qui veulent une justice qui tue, ceuxlà sont animés par une double conviction: qu'il existe des hommes totalement coupables, c'est-à-dire des hommes totalement responsables de leurs actes, et qu'il puisse y avoir une justice sûre de son infaillibilité au point de dire que celui-là peut vivre et que celui-là doit mourir.

À cet âge de ma vie, l'une et l'autre affirmations me paraissent également erronées. Aussi terribles, aussi odieux que soient leurs actes, il n'est point d'hommes en cette terre dont la culpabilité soit totale et dont il faille pour toujours désespérer totalement. Aussi prudente que soit la justice, aussi mesurés et angoissés que soient les femmes et les hommes qui jugent, la justice demeure humaine, donc faillible [...]. Parce qu'aucun homme n'est totalement responsable, parce qu'aucune justice ne peut être totalement infaillible, la peine de mort est moralement inacceptable [...] (Robert Badinter, garde des Sceaux, AN, p. 1142)

\section{La formulation des croyances implicites de l'adversaire}

L'argumentation abolitionniste s'attache à donner d'abord une formulation parfaitement explicite aux croyances «secr[ètes] » et «inavoué[es] » qui fondent la «logique de la guillotine» (Gisèle Halimi, AN, p. 1159). Dans l'extrait (3), Robert Badinter énonce les conditions d'acceptabilité - ou d'admissibilité - de l'application de la peine de mort. La reconstruction de la «logique de la guillotine» s'effectue de la manière suivante: le ministre attribue à ses adversaires («Ceux qui veulent une justice qui tue») des états mentaux («ceux-là sont animés par une double conviction...») et des contenus propositionnels $\mathrm{p}$ et q («il existe des hommes totalement coupables, c'est-à-dire des hommes totalement responsables de leurs actes » (p) et «il p[eut] y avoir une justice sûre

13. C. Perelman, 1990, Éthique et droit, Bruxelles, Éditions de l'Université de Bruxelles, p. 337 . 
de son infaillibilité au point de dire que celui-là peut vivre et que celui-là doit mourir» (q)). On pourrait représenter les choses ainsi :

Croyance (p): «il existe des hommes totalement coupables, c'est-à-dire des hommes totalement responsables de leurs actes».

Croyance (q) : «il p[eut] y avoir une justice sûre de son infaillibilité au point de dire que celui-là peut vivre et que celui-là doit mourir».

Croyance (r): «la justice qui tue le criminel» est «acceptable».

Si l'on suit la «logique de la guillotine»: Croyance (p) et Croyance (q) justifient Croyance (r).

Il s'agit, on le voit, d'établir un lien indissoluble entre, d'une part, la volonté d' «une justice qui tue» et, d'autre part, la «double conviction» évoquée. Si l'on veut prendre l'adversaire au piège de sa propre logique, cette dernière doit d'abord être présentée de la manière la plus implacable possible. Dans cet esprit, R. Badinter lui donne un maximum de simplicité, de transparence et, pourrait-on dire, de lisibilité.

Ces croyances traduisent, dit R. Badinter, une «certaine conception de l'homme et de la justice». Arrêtons-nous un instant sur la qualification des «hommes» (Croyance (p)) en examinant brièvement le syntagme «des hommes totalement coupables ». L'adverbe totalement opère un travail de figement sur l'adjectif coupables et lui confère une dimension monolithique. Une telle conception se heurte à la distinction qu'établit John Searle entre les «faits bruts» et les «faits institutionnels» dont relèvent, par exemple, les procès ${ }^{14}$. Dans une telle optique, la culpabilité est bien la résultante d'un fait institutionnel: elle consiste, selon les termes de J. Searle, en l'imposition d'un nouveau statut à un individu par une instance humaine habilitée pour cela - par exemple un tribunal de cour d'assises. La culpabilité d'un individu n'est ainsi pas un fait brut, puisqu'elle doit être prononcée par un juge lors d'un acte de langage de type déclaratif ${ }^{15}$. S'il comporte bien une double dimension à la fois épistémologique - dire la vérité - et pragmatique - entrainer une sanction - un tel acte de langage ne saurait prétendre qualifier l'individu dans son essence: ce dernier sera toujours déclaré coupable de quelque chose, d'un ou plusieurs faits ponctuels. L'adverbe totalement, en revanche, tend à fixer ontologiquement la culpabilité qui devient dès lors une propriété essentielle et non plus accidentelle de l'individu. Le syntagme des hommes totalement coupables

14. J. Searle, 1995, The Construction of Social Reality, Londres, Penguin, p. 50.

15. E. Danblon, 2002, Rhétorique et rationalité, Bruxelles, Éditions de l'Université de Bruxelles, p. 87-99. 
souffre ainsi d'une tension insupportable entre le nom et sa qualification: les hommes, dans une perspective humaniste, ne sauraient être porteurs d'une culpabilité irréversible et définitive.

Lorsque R. Badinter en vient à la seconde croyance, celle concernant la justice (Croyance (q)), il vise un effet similaire. La notion d'infaillibilite est porteuse, dans la culture judéo-chrétienne, de fortes résonances religieuses. Dans le discours abolitionniste, la «justice» dont il est question n'est cependant plus la justice d'un Dieu lointain et redouté. Au contraire: c'est, dans la France laïque de la fin du vingtième siècle, la justice en tant qu'institution prise en charge par des hommes qui est visée ici. Dans une telle optique, lorsque R. Badinter évoque une «justice sûre de son infaillibilité au point de dire que celui-là peut vivre et que celui-là doit mourir», il cherche à créer, une fois encore, un effet de décalage. Ce décalage se situe entre une notion, la justice, redescendue du niveau divin au niveau humain, et une propriété, l'infaillibilité, qui, dans l'univers de croyances de la société française moderne, ne peut plus s'appliquer à grand chose.

\section{Une réfutation de la logique de la guillotine}

L'attribution de croyances à l'adversaire, on le voit, est loin d'être une opération innocente: la formulation même du contenu propositionnel tend à présenter ces dernières comme intenables. Dans la suite de son argumentation, $\mathrm{R}$. Badinter opère pour ainsi dire le retournement des croyances adverses. Il semble s'agir ici de l'application d'une fonction logique fort simple: celle de négation. Cette dernière, on le sait, envoie la valeur de vérité d'une proposition sur la valeur de vérité opposée: $s i \mathrm{p}=1$, alors $\neg \mathrm{p}=0$, et inversement. Lorsque Badinter affirme qu' «à cet âge de [sa] vie, l'une et l'autres affirmations [lui] paraissent également erronées », il annonce la négation des croyances adverses auxquelles il vient de donner un contenu propositionnel. Ces propositions, on s'en souvient, contenaient toutes deux des expressions à valeur de quantification existentielle: les antiabolitionnistes, selon R. Badinter, croient qu' «il existe des hommes totalement coupables [...] et qu'il puisse y avoir une justice sûre de son infaillibilité...» (je souligne). La négation va dès lors, on s'en doute, venir contester l'affirmation d'existence des individus et objets dénotés.

La force de l'argumentation, dans cet esprit, est d'éviter un double écueil: d'une part celui de l'angélisme et, d'autre part, celui du désenchantement. Nier l'existence d'hommes «totalement coupables» ne doit pas revenir, dans une perspective abolitionniste, à minimiser l'horreur des crimes commis. Pour contrer anticipativement une telle objection, R. Badinter souligne le caractère «terrible» et «odieux» des «actes» criminels. Cependant, il prend bien soin, dans la structure syntaxique même de sa phrase, de dissocier ces actes de leurs 
agents. La proposition concessive en aussi... que empêche le passage mécanique de la sphère de l'action à celle de l'être. Cette concession nous parait importante rhétoriquement: elle contribue en effet à débarrasser l'orateur abolitionniste d'un éthos humaniste naïf, aveuglé par sa foi en l'homme. Dès lors, la proposition assertive $\neg$ p qui suit («il n'est point d'hommes en cette terre dont la culpabilité soit totale et dont il faille pour toujours désespérer totalement»), presque exactement contraire à $\mathrm{p}$, échappe en partie à l'accusation d'angélisme.

Tournons-nous à présent vers la seconde croyance implicite du discours adverse, à savoir celle qui postule l'existence d'une «justice sûre de son infaillibilité». La contestation d'une telle croyance présente certains risques pour l'orateur. Certes, il peut se targuer d'être réaliste... mais ce réalisme critique ne doit pas apparatre comme un désenchantement, ou, pire, comme une attitude de défiance face à l'autorité judiciaire! Dans cette optique, la concessive en aussi... que a pour rôle de souligner les qualités réelles de l'institution judiciaire (la prudence) et de ses acteurs (la mesure et l'angoisse au moment de juger). Elle évite dès lors à l'orateur qualifiant la justice d'«humaine», donc de «faillible» de passer pour un désenchanté qui, à la limite, en viendrait à identifier cette dernière à une loterie purement arbitraire.

Il nous reste à élucider la manière exacte dont l'argumentation abolitionniste réfute le raisonnement adverse. Il s'agit, pour R. Badinter, de prendre l'adversaire au piège de sa propre logique, en traitant $\mathrm{p}$ et $\mathrm{q}$ moins comme les prémisses possibles d'une adhésion à la peine de mort que comme ses implications inévitables. La première étape consiste, dans cet esprit, à montrer à l'adversaire qu'accepter l'application de la peine de mort l'engage de manière absolument contraignante à accepter également $\mathrm{p}$ et $\mathrm{q}$ :

1) Si la peine de mort est acceptable (r), alors c'est «qu'il existe des hommes totalement coupables» (p) et une «justice sûre de son infaillibilité au point de dire que celui-là peut vivre et que celui-là doit mourir» (q).

La formulation même de $\mathrm{p}$ et de $\mathrm{q}$, on vient de le voir, rend ces propositions difficilement tenables. La seconde étape consiste en leur négation explicite:

2) Or «il n'est point d'hommes en cette terre dont la culpabilité soit totale [...]» $(\neg \mathrm{p})$ et «la justice demeure humaine, donc faillible $[\ldots] »(\neg \mathrm{q})$.

La dernière étape de la réfutation est décisive. À ce stade, l'inacceptabilité des conséquents $\mathrm{p}$ et $\mathrm{q}$ se voit transférée sur $\mathrm{r}$. Les propositions $\neg \mathrm{p}$ et $\neg \mathrm{q}$ deviennent alors les fondements du refus de la peine de mort. Le raisonnement pourrait se laisser saisir ainsi : $\mathrm{r}$ engage nécessairement à $\mathrm{p}$ et à $\mathrm{q}$ (étape 1 ); $\mathrm{p}$ et q sont inacceptables (étape 2); parce que p et q sont inacceptables, $\mathrm{r}$ aussi est inacceptable: 
3) «Parce qu'aucun homme n'est totalement responsable» ( $\neg$ p) et «parce qu'aucune justice ne peut être totalement infaillible,» $(\neg$ q) «la peine de mort est moralement inacceptable» $(\neg \mathrm{r})$.

Les croyances latentes portées au grand jour ne sont pas réfutées en tant qu'antécédents possibles, mais bien en tant qu'implications nécessaires de tout discours qui voudrait établir l'acceptabilité d'une «justice qui tue le criminel». Après une telle réfutation, la question de savoir si l'usage de la peine de mort pourrait se justifier autrement que par la croyance en la culpabilité irréversible de certains hommes ou en l' «infaillibilité» de la justice n'est plus vraiment pertinente. Il ne saurait plus s'agir, pour les antiabolitionnistes, de partir à la recherche d'autres prémisses pour justifier leur point de vue. De toute façon, leur adhésion à la peine capitale les engage, quelle que soit sa nature, à reprendre à leur compte des croyances dont l'argumentation de R. Badinter a cherché à faire ressortir, outre la non-satisfaction empirique, le caractère «moralement insupportable».

Le parcours proposé ici vient étayer l'hypothèse d'une divergence notable entre les discours antiabolitionnistes et abolitionnistes tant au niveau de la qualification de l'objet soumis à délibération que de la direction que prend l'argumentation. Les orateurs anti-abolitionnistes apparaissent privilégier de manière assez nette les critères très typiquement délibératifs de l'utile et du nuisible lorsqu'ils se penchent sur l'objet de la délibération; ils travaillent ainsi prioritairement en aval d'une décision parlementaire dont ils jaugent les conséquences dans l'avenir. Les abolitionnistes, en revanche, travaillent préférentiellement en amont d'une décision parlementaire dont ils cherchent à établir les fondements. Leur argumentation tend ainsi plutôt à déterminer ce qu'il est juste ou au contraire injuste de faire dans et par rapport à un certain «contexte de valeurs et de normes ${ }^{16}$. On a ainsi, selon la posture discursive adoptée, une dominante délibérative plus ou moins marquée. Cela éclaire, je crois, le combat pour la légitimité au sein de l'institution parlementaire et, plus généralement, la controverse entre abolitionnistes et antiabolitionnistes.

16. C. Perelman, 1990, ouvr. cité, p. 337. 